\title{
Circadian rhythms and leaf characteristics of subalpine Eucalyptus pauciflora: The effects of environmental exposure on photosynthetic machinery
}

\author{
Christine Mauger, Julia Hammer, Chen Liang, Cameron \\ McArthur, Angela Stoddard
}

\section{Abstract}

This study explored whether snow gums (Eucalyptus pauciflora) in a subalpine region use circadian rhythms for photosynthesis and whether age and exposure affect photosynthetic machinery. Fv/Fm, SPAD and specific leaf area (SLA) were measured in individual leaves to assess variation in leaf traits. $\mathrm{Fv} / \mathrm{Fm}$ was not significantly different between exposed and sheltered trees, but old leaves had significantly higher Fv/Fm values than young leaves. SPAD was higher in exposed trees and old leaves had significantly higher chlorophyll content. SLA was significantly lower in old leaves, and sheltered leaves had significantly higher SLA than exposed leaves. Increase in SLA with exposure was greater in young leaves. Branches from sheltered and exposed E. pauciflora were harvested then placed in light and dark treatments. Stomatal conductance was tested at various times to determine if leaf stomata were opening without access to sunlight. At the time of this experiment, there had been no previous known studies on circadian rhythms in E. pauciflora. Conductance rates were higher in the light treatment, and across this treatment it was higher at $11 \mathrm{am}$ than at night. There was no effect of exposure on conductance rates in the light treatment. In the dark treatment, exposed leaves had lower conductance rates than sheltered leaves. For sheltered and exposed leaves, conductance was greater at dawn than at $11 \mathrm{pm}$ and greater again at 11 am than at dawn, suggesting E. pauciflora do use circadian rhythms for photosynthesis. 


\section{Introduction}

Photosynthesis is the conversion of light energy to chemical energy, stored as sugars or other compounds in plants. For photosynthesis to take place, the plant undergoes gas exchange, which is controlled by the stomata within the leaf's epidermis. In subalpine to alpine areas such as the Australian Snowy Mountains low temperatures occur during the spring snow melt. There is a short growing season with water and light availability restricted. A plant's ability to undergo photosynthesis is dependent on resource availability, and so in these areas plants need to adapt to deal with such stresses. Most organisms do not respond specifically to sunrise, but do await the dawn. When external time cues are absent, many of these diurnal rhythms continue, indicating there is an endogenous biological circadian clock (McClung 2006). Circadian rhythms demonstrate an approximate 24-hour period or cycle. They are generated internally within the organism, and continue under free-running conditions like constant light or darkness, and temperature (de Dios et al. 2009). The circadian clock influences gas exchange in a plant by preparing for dawn and dusk cycles (de Dios et al. 2009) and is thought to regulate a large number of plant processes. Circadian rhythms assist plant growth, meaning they can give plants a competitive advantage. This has been demonstrated in the model plant Arabidopsis thaliana. Dodd et al. (2005) found that in wild type and mutants of $A$. thaliana, plants that had a clock period matching the environment contained more chlorophyll. They were also able to fix more carbon, as well as grow faster and survive better, showing that circadian control gives plants an advantage. The diurnal variation in photosynthetic gas exchange is of interest to botanists, ecologists, conservationists and climate modellers, who seek to understand the effect future changes in climate may have on ecosystems (de Dios et al. 2009). Stomatal conductance is a measurement of the rate that carbon dioxide enters or water vapour exits the stomata. Because stomata are open during photosynthesis, stomatal conductance can be used as a proxy to measure photosynthetic activity. Typically, stomatal conductance increases with light up until around midday. In the middle of the day, the demand for water from the atmosphere increases, and to avoid unnecessary water loss, stomatal conductance declines (de Dios et al. 2009).

Leaf morphology can also affect photosynthetic characteristics. Leaf characteristics may vary depending on exposure, orientation and age in ways that alter carbon gain patterns. Specific leaf area (SLA) is the ratio 
of leaf area to dry mass, measured in $\mathrm{cm}^{2} / \mathrm{g}$. Typically, thicker leaves have a lower SLA and have more sclerenchyma. Sclerenchyma is a plant tissue that provides strength, making a plant hardy. Thinner leaves have higher SLA with more mesophyll cells, which are specialised for photosynthesis (Steane et al. 2015). SPAD is a measurement of chlorophyll content within leaves and is thus linked to photosynthetic ability. The amount of sunlight that a leaf absorbs is a function of the chlorophyll; therefore, chlorophyll content affects photosynthetic potential (Gitelson et al. 2003; Curran et al. 1990; Filella et al. 1995). Fv/Fm is a measurement of chlorophyll fluorescence and an indicator of photosystem health. Chlorophyll fluorescence is commonly used to measure plant stress and is linked to photosynthetic efficiency (Patankar et al. 2013).

E. pauciflora is the dominant species found at the tree line in the Australian Alps (Brookhouse et al. 2008) and is a keystone species occurring in the Kosciuszko National Park. While there have been some studies done on circadian rhythms in Eucalypt species (de Dios et al. 2013), there has been none on E. pauciflora. Given future climate change predictions for the subalpine and alpine regions, it is important to understand how this species' photosynthetic machinery works in varying conditions.

The aim of this study was to see if stomatal conductance varied over a 24-hour period, and if it was dependant on the leaf's position, its exposure and age in subalpine E. pauciflora without any environmental cues. Our main hypothesis with the above factors considered was that exposed snow gums would show less circadian rhythm regulation, in terms of stomatal conductance, than sheltered snow gums.

We assessed variation in leaf level gas exchange over a 24-hour period in E. pauciflora. SLA, chlorophyll content (SPAD) and the efficiency of photosynthetic machinery $(\mathrm{Fv} / \mathrm{Fm})$ were assessed. In addition to time of day, we also considered the amount of variation within and between plants, looking at the age of the leaves and their position on the plant. When looking at variation in leaf traits, we hypothesised Fv/Fm would be lower in exposed plants and that young leaves would have higher Fv/Fm. We hypothesised that age and exposure would affect chlorophyll content (SPAD) with it being lower in exposed leaves, and lower in older leaves. Our last hypothesis was that SLA would be lower in exposed leaves. 


\section{Methods}

\section{Study site}

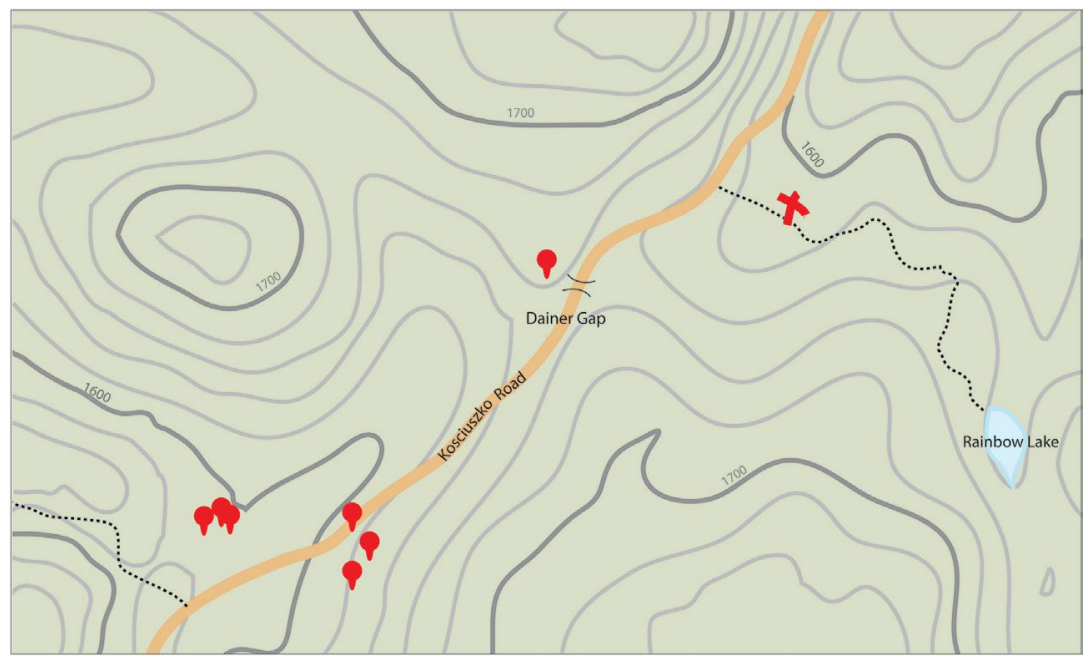

Figure 1: Site map showing sampling sites. Sheltered branches and leaves were harvested on the Rainbow Lake path in subalpine woodland and are marked with $X(n=5)$. Exposed trees that were sampled along the road between Charlotte Pass and Rainbow Lake are marked with red pins $(\mathrm{n}=7)$.

Source: Created by the authors in GPS visualiser (www.gpsvisualizer.com).

Sheltered branches were collected close to the Rainbow Lake walking trail $\left(-36.371114^{\circ} S, 148.479931^{\circ} \mathrm{E}\right)$ in Kosciuszko National Park, New South Wales, Australia. Selected exposed trees were single standing so they were fully exposed to environmental conditions (Figure 1).

\section{Circadian rhythm measurements}

One branch from each sheltered tree $(n=5)$ and exposed tree $(n=7)$ was collected to be used for the light and dark treatments. The branches had healthy leaves and were of a size that could be broken to create two branches. Branches were numbered (1 to 5 and 6 to 12) and stems were cut far down. Branches were individually wrapped in wet paper towel and placed in a plastic bag containing water. Samples were taken to the lab to prepare for stomatal conductance measurements in light and dark treatments. A bucket was filled halfway with cold tap water. Each branch 
was placed in the water, where a cut was made below water level to reduce the risk of embolisms, caused by allowing air to enter the vascular tissue. While still below the water, the branch was placed into a zip-lock bag and labelled with tree number, and then into a tall cup so it could stand up straight. If the bottom of the branch came out of water during movement, it was cut again below the water level. Once all branches were prepared for treatment and placed in cups, they were randomly allocated into two groups (for light and dark treatment) with one branch from each tree put into each group. Light treatment branches were put under constant artificial lighting (JB HPS400 N4208 grow lamp). Dark treatment branches were placed in a constantly dark, windowless room so light was unable to enter.

Each branch had a single leaf that was marked with tape and tree number. Branches were left in treatment conditions for at least eight hours before conductance measurements were taken. Stomatal conductance and temperature measurements were taken using the Decagon SC-1 Leaf Porometer for marked leaves at $11 \mathrm{pm}$ and then $5 \mathrm{am}$ and $11 \mathrm{am}$ the following morning. Measurements of dark treatment branches were conducted using a green light setting on a head torch, to avoid affecting results.

\section{Leaf trait measurements}

Leaf samples were taken back to the lab for Fv/Fm, SPAD and SLA measurements. Four individual leaves were taken from the same trees where branches were removed, with a combination of young, old, northfacing and south-facing leaves (old = older than last years' growth, young = last years' growth). Selected sheltered trees were those close together, with the aim of minimising differences in exposure levels. Leaves were numbered and placed into a zip-lock bag. Fv/Fm was measured using a Plant Efficiency Analyser. Dark adaptation was conducted for 20 minutes on each individual leaf, using leaf clips before receiving a light flash to centre of leaf and measuring Fv/Fm. Chlorophyll content was measured with a Konica Minolta Chlorophyll Meter (SPAD-502) in SPAD units as an average of three measurements for each leaf. Leaves were photographed and had leaf area measured using image analysis with ImageJ. Leaves were dried overnight and each individual leaf had SLA $\left(\mathrm{cm}^{2} / \mathrm{g}\right)$ measured using an electronic balance. SLA is calculated by dividing the area of the leaf in $\mathrm{cm}^{2}$ by the dry leaf mass in grams. 


\section{Statistical analyses}

The data were tested using analysis of variance (ANOVA) to look at the effects age and exposure had on SLA, Fv/Fm, SPAD and also on stomatal conductance.

\section{Results}

The ANOVA (Table 1) yielded the following results: old leaves have significantly higher $(P<0.001) \mathrm{Fv} / \mathrm{Fm}$ than young leaves but both are quite healthy. Fv/Fm was slightly higher in old exposed leaves compared to old sheltered leaves (Figure 2a). Photosynthetic machinery efficiency ( $\mathrm{Fv} / \mathrm{Fm})$ was not significantly different $(P=0.673)$ between exposed and sheltered trees, or between age and exposure $(P=0.535)$. Old leaves have significantly higher $(P<0.001)$ chlorophyll content (SPAD). When comparing sheltered and exposed sites, SPAD was higher, but not significantly $(P=0.200)$, in leaves from exposed trees (Figure $2 \mathrm{~b}$ ). Old leaves have significantly lower $(P<0.001)$ SLA than young leaves, and sheltered leaves have significantly higher SLA than exposed leaves $(P<0.001)$. The increase in SLA with exposure is also significantly $(P=0.010)$ greater in young leaves (Figure $2 c$ ).

Table 1: ANOVA summary table.

\begin{tabular}{|l|c|c|c|}
\hline Source & SLA & Fv/Fm & SPAD (chlorophyll) \\
\hline Age & $* * *$ & $* * *$ & $* * *$ \\
\hline exposure & $* * *$ & $\mathrm{~ns}$ & $\mathrm{~ns}$ \\
\hline Age exposure & $*$ & $\mathrm{~ns}$ & $\mathrm{~ns}$ \\
\hline
\end{tabular}

Note: ${ }^{\star} P<0.05,{ }^{\star \star} P<0.01,{ }^{* \star} P<0.001$, ns $=$ not significant.

Source: Authors' data. 
a)

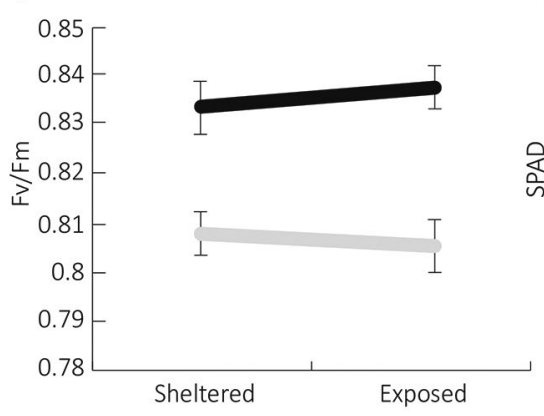

b)

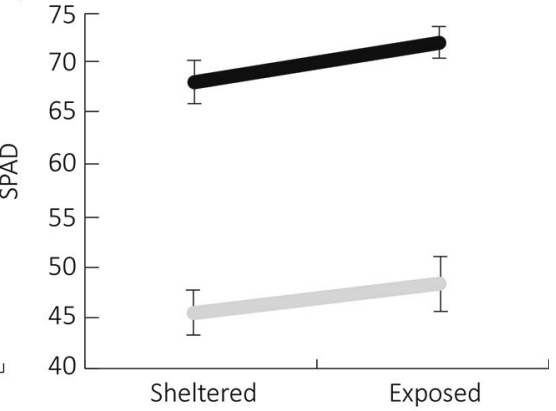

c)

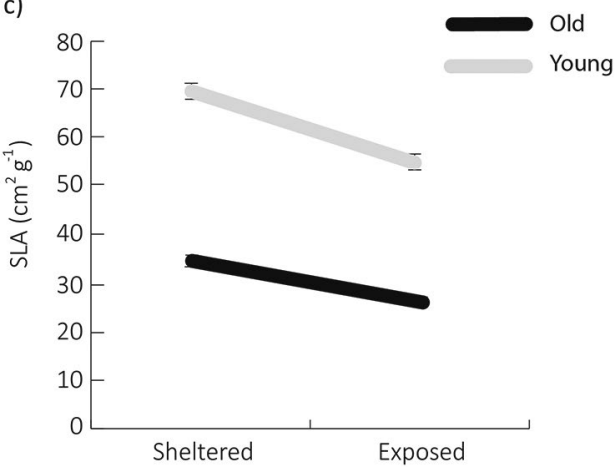

Figure 2: a) Comparison of average Fv/Fm in young and old leaves within sheltered and exposed sites, b) Comparison of average SPAD in young and old leaves within sheltered and exposed sites, C) Comparison of average SLA in young and old leaves within sheltered and exposed sites. Note: Error bars are standard error.

Source: Authors' data.

Stomatal conductance of sheltered leaves (Figure 3a) increased overnight under the dark treatment, nearly doubling from a mean of $53 \mathrm{mmol} \mathrm{m}^{-2} \mathrm{~s}^{-1}$ at $11 \mathrm{pm}$ to a mean of $96 \mathrm{mmol} \mathrm{m}^{-2} \mathrm{~s}^{-1}$ at $11 \mathrm{am}$. Stomatal conductance rates of exposed leaves (Figure 3b) increased overnight under dark treatment with the mean conductance starting at $40 \mathrm{mmol} \mathrm{m}^{-2} \mathrm{~s}^{-1}$ at $11 \mathrm{pm}$ and 74 $\mathrm{mmol} \mathrm{m} \mathrm{m}^{-2} \mathrm{~s}^{-1}$ at $11 \mathrm{am}$.

ANOVA results also show that plants in the dark treatments had significantly $(P=0.014)$ lower conductance rates in exposed leaves than in sheltered leaves. For both exposures in the dark, ANOVA results show conductance was greater at dawn than at $11 \mathrm{pm}$, and significantly $(P=0.024)$ greater again at 11 am compared to dawn. 
a)
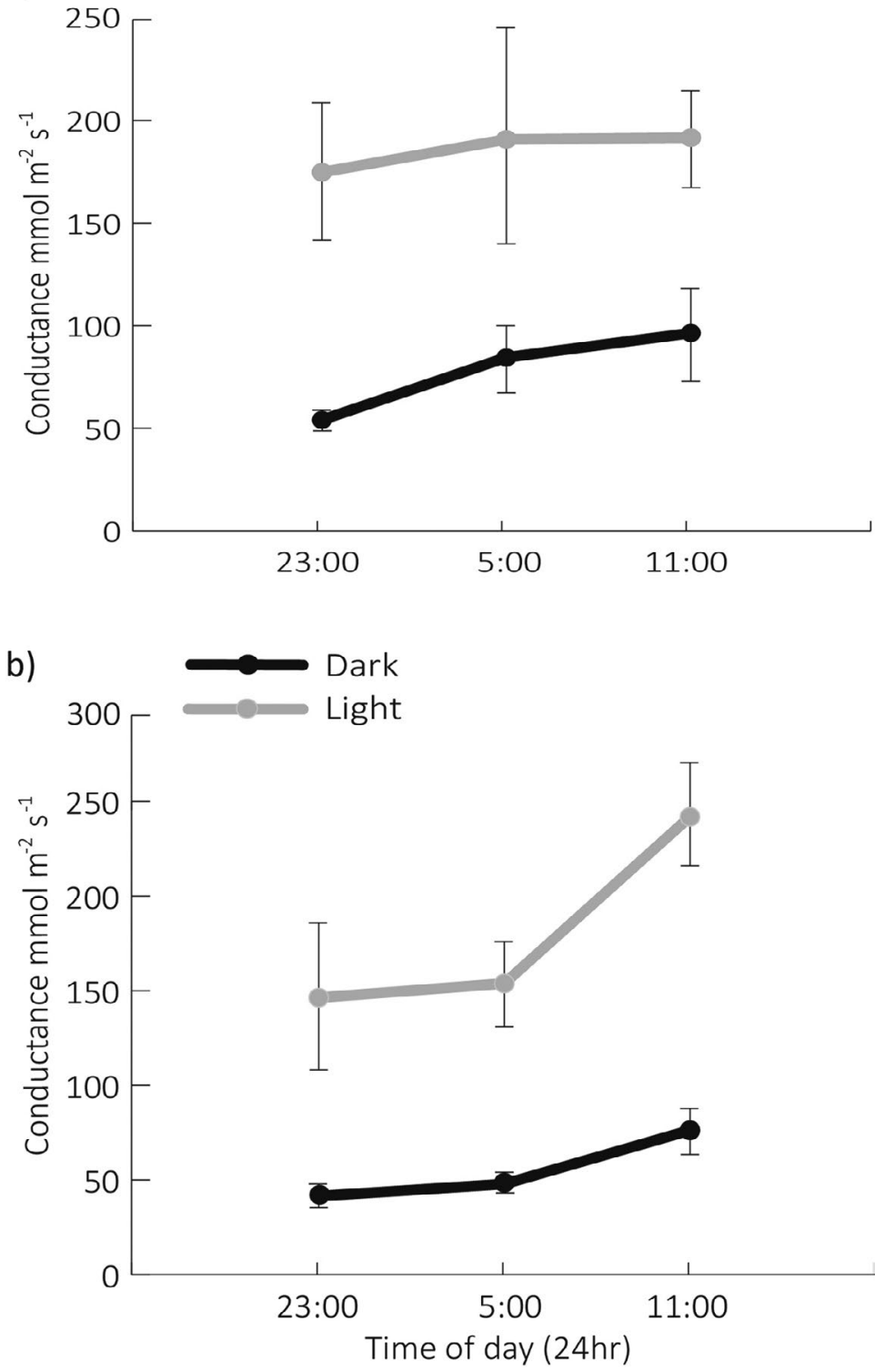

Figure 3: a) Mean conductance measurements of sheltered leaves under continuous dark and light treatments. Conductance was measured at 23:00, 05:00 and 11:00 hours, b) Mean conductance measurements of exposed leaves under continuous dark and light treatments. Conductance was measured at 23:00, 05:00 and 11:00 hours.

Note: Error bars are standard error.

Source: Authors' data. 
Under the light treatment stomatal conductance increased from $11 \mathrm{pm}$ to $5 \mathrm{am}$ (by $16 \mathrm{mmol} \mathrm{m}^{-2} \mathrm{~s}^{-1}$ ), but not from 5 am to $11 \mathrm{am}$ for sheltered leaves. The conductance rate was much higher for sheltered leaves in the light treatment (mean 174-190 $\mathrm{mmol} \mathrm{m}^{-2} \mathrm{~s}^{-1}$ ); however, dark sheltered leaves showed a greater increase in stomatal conductance over time.

When looking at light and exposure effects, results from the ANOVA showed that conductance was significantly $(P<0.001)$ higher in the light, and across light treatments it was significantly higher at 11 am than at night $(P=0.035)$. Under the light treatment, stomatal conductance of exposed leaves increased overnight from a mean of $145 \mathrm{mmol} \mathrm{m}^{-2} \mathrm{~s}^{-1}$ at $11 \mathrm{pm}$ to a mean of $240 \mathrm{mmol} \mathrm{m}^{-2} \mathrm{~s}^{-1}$ at $11 \mathrm{am}$.

Light treatment leaves from exposed trees appeared to capitalise more on light around midday than those from sheltered sites; however, when considering just those plants in the light treatment, there is no significant effect of exposure $(P=0.642)$, or period $(P=0.156)$ on conductance.

\section{Discussion}

\section{Leaf traits}

We hypothesised $\mathrm{Fv} / \mathrm{Fm}$ would be lower in exposed plants, with the need of investing energy into sclerenchyma over mesophyll cells, and that young leaves would have higher Fv/Fm. The ANOVA (Table 1) results showed old leaves have significantly higher $(P<0.001) \mathrm{Fv} / \mathrm{Fm}$ than young ones but both are quite healthy. A leaf with $\mathrm{Fv} / \mathrm{Fm}$ above 0.8 is considered a healthy leaf. This was not expected as typically younger leaves are better at photosynthesising, but it may be due to our selection methods where old was considered to be anything older than the previous year's growth. The ANOVA shows that photosynthetic machinery efficiency $(\mathrm{Fv} / \mathrm{Fm})$ was not significantly different $(P=0.673)$ between exposed and sheltered trees, or between age and exposure $(P=0.535)$, going against our hypothesis.

We hypothesised that age and exposure would affect chlorophyll content (SPAD) with it being lower in exposed leaves, and lower in older leaves. The ANOVA (Table 1) showed that old leaves in fact have significantly higher $(P<0.001)$ chlorophyll content (SPAD), going against our hypothesis. This is likely to be that energy is going into plant structural growth. When comparing sheltered and exposed sites, SPAD was higher 
but not significantly $(P=0.200)$ in leaves from exposed trees (Figure $2 b$ ), also going against our hypothesis. SPAD may be lower in sheltered trees as less light is able to reach the leaves as they are in close proximity to each other so chlorophyll content is lower.

The ANOVA (Table 1) showed that old leaves have significantly lower $(P<0.001)$ SLA than young leaves and that sheltered leaves have significantly higher SLA than exposed leaves $(P<0.001)$. The increase in SLA with exposure is also significantly $(P=0.010)$ greater in young leaves. These results support our hypothesis that SLA is lower in exposed leaves. High SLA is typical of thinner or less dense, younger leaves. An explanation for lower SLA in exposed trees is that more extreme environmental conditions may have led to the development of more hardy structural tissues for leaf protection.

\section{Circadian rhythm}

Our main hypothesis was that exposed snow gums would show less circadian rhythm regulation, in terms of stomatal conductance, than sheltered snow gums. Leaves from the dark treatment (both exposed and sheltered trees) became more active, with stomata conductance increasing from $11 \mathrm{pm}$ to $11 \mathrm{am}$, indicating there is circadian rhythm in E. pauciflora. ANOVA results show that in plants in the dark treatments there was significantly $(P=0.014)$ lower conductance in exposed leaves than in sheltered leaves. These results support our hypothesis that exposed snow gums would show less circadian rhythm, in terms of stomatal conductance, than sheltered snow gums. Light treatment leaves from exposed trees appeared to capitalise more on light around midday than those from sheltered sites. However, when considering just those plants in the light treatment, there is no significant effect of exposure $(P=0.642)$, or period $(P=0.156)$ on conductance; when looking at circadian rhythm, it is the dark treatment that needs to be considered.

Many previous studies looking at circadian rhythms in plants show clear diurnal patterns in photosynthesis and respiration. Conductance peaking at midday and lowering around midnight in the dark treatment indicates the use of circadian rhythm (Patankar et al. 2013). Stomatal conductance rates were higher in leaves from sheltered trees at the measurement times of $11 \mathrm{pm}$ and $5 \mathrm{am}$ for both treatments compared to leaves from exposed trees (Figures $3 \mathrm{a}$ and $3 \mathrm{~b}$ ). The $11 \mathrm{pm}$ measurement was also higher in the dark treatment for sheltered leaves than exposed. Interestingly, the 
light treatment leaves from exposed trees appeared to capitalise more on light around midday than those from sheltered sites with a difference in $50 \mathrm{mmol} \mathrm{m}^{-2} \mathrm{~s}^{-1}$.

For a circadian rhythm to have adaptive benefit, there must be consistency between average days. Circadian rhythms persist although external cues are absent. The large increase in stomatal conductance for exposed trees in the light treatment could be due to midday being the time there is the most solar light available for energy production and they are adapted to utilising the light, being fully exposed to it.

Generally, photosynthetic gas exchange is strongly driven by the environment. However, many studies have now provided evidence that circadian regulation plays a large, important role in many plant species (de Dios et al. 2009). This could explain why the conductance rate in exposed trees in the light treatment was so high. This could also explain why the conductance rates for the sheltered and exposed leaves in the light treatment only had a slight increase between $11 \mathrm{pm}$ and $5 \mathrm{am}$. Being constantly in front of a light treatment, they had reached the optimal conductance rate, and the circadian clock at that time did not allow for more stomata to open in the risk of losing water.

In subalpine regions, there are many stresses to plants and so they may have limited opportunity to photosynthesise each day. Opening stomata before light hits the plant gives them a competitive advantage against others, limiting water loss that may occur from evaporation during the day.

\section{Further studies}

Sheltered leaves and branches that were harvested came from an area (Rainbow Lake) that had been burnt in the 2003 fires. Exposed leaves came from an unburnt area. It would be interesting to test if some of these results were due to rehabilitation. When looking at this factor, it would be important to look both at exposed trees from a similar area and at sheltered trees from an unburnt area for comparison. Testing leaves from the east and west sides of trees may also give different results.

If this study was to be replicated, it would be ideal to have the same sample number for exposed and sheltered sites as the numbers were uneven $(\mathrm{n}=7$ and $n=5$ ) in this experiment, which could possibly skew the results. It is 
important to note that there were a number of people working on this project with a handover, possibly leading to variations in procedures and measurements causing possible human error.

As this was a study with a small sample size, at one time of the year, it would be ideal to test if the same trees (sheltered and exposed) went through any seasonal changes in relation to stomatal conductance and if the quality of photosynthetic machinery varied. If these factors affect growth, survival and competitive advantage, a longitudinal study would provide data either proving or disproving this, and it may vary during seasons.

Seasonal patterns of plant physiology recorded at midday in the Northern Hemisphere show that photosynthesis is often lowest right after snow melt in spring. It reaches its peak around the middle of the growing season in mid-summer, and decreases again towards autumn (Defoliart et al. 1988; Starr et al. 2000, 2008). Around autumn is when leaves start hardening and the health of the leaves deteriorate (Oberbauer and Starr 2002). This is interesting as the results of this study show some of the older leaves tested had greater photosynthetic efficiency and greater chlorophyll content. It would be interesting to see if this is a general seasonal pattern that also occurs in the Snowy Mountains for other evergreen Eucalypt species, as this was a one-time experiment conducted at the start of summer. Whilst undergoing statistical analysis for SLA, there was one outlier removed, which was of young age and exposed. Removing this outlier did not affect the pattern of results.

\section{Conclusions}

As E. pauciflora is a keystone species of the Kosciuszko National Park, understanding the diurnal physiology of snow gums is important and can help when considering conservation projects, including producing models. It would be particularly helpful for the use of predicting and understanding the effects of climate change on the Australian Snowy Mountain flora. Climate change may lead to a longer growing season, with warmer summers. Change in temperatures may induce a shift in the composition of plant communities in the region.

In relation to global climate change predictions in the Snowy Mountains of Australia, an increase in ambient temperature will likely result in greater water loss through stomatal conductance. This will affect plants, ability to 
photosynthesise and limit their growth. Plants that use circadian rhythm to control stomatal conductance, opening stomata during hours of darkness, will have a competitive advantage over those that do not. This allows those using circadian rhythms to capitalise on morning light without losing too much water. However, without endogenous control to close the stomata and stop photosynthesising during the hottest time of the day, plants may lose large amounts of water and fall victim to heat stress.

\section{Acknowledgements}

Thanks to our project supervisor Adrienne Nicotra, and to Sarah Stock, Hannah Zurcher, Ming-Dao Chia and Tess Walsh-Rossi for a smooth project handover and for supplying data for sheltered leaves/branches.

\section{References}

Brookhouse M, Lindesay J, Brack C (2008) The potential of tree rings in Eucalyptus pauciflora for climatological and hydrological reconstruction. Geographical Research 46: 421-34. doi.org/10.1111/ j.1745-5871.2008.00535.x

Curran PJ, Dungan JL, Gholz HL (1990) Exploring the relationship between reflectance red edge and chlorophyll content in slash pine. Tree Physiology 7: 33-48. doi.org/10.1093/treephys/7.1-2-3-4.33

de Dios VR, Diaz-Sierra R, Goulden ML, Barton CVM, Boer MM, Gessler A, Ferrio JP, Pfautsch S, Tissue DT (2013) Woody clockworks: Circadian regulation of night-time water use in Eucalyptus globulus. New Phytologist 200: 743-52. doi.org/10.1111/nph.12382

de Dios VR, Hartwell J, Hall A (2009) Ecological implications of plants' ability to tell the time. Ecology Letters 12: 583-92. doi.org/10.1111/ j.1461-0248.2009.01295.x

Defoliart L, Griffith M, Chapin FS, Jonasson S (1988) Seasonal patterns of photosynthesis and nutrient storage in Eriophorum vaginatum L., an arctic sedge. Functional Ecology 2: 185-94. doi. org/10.2307/2389694 
Dodd AN, Salathia N, Hall A, Kévei E, Tóth R, Nagy F, Hibberd JM, Millar AJ, Webb AA (2005) Plant circadian clocks increase photosynthesis, growth, survival, and competitive advantage. Science 309(5734): 630-3. doi.org/10.1126/science.1115581

Filella I, Serrano I, Serra J, PeñuelasJ (1995) Evaluating wheat nitrogen status with canopy reflectance indices and discriminant analysis. Crop Sci 35: 1400-5. doi.org/10.2135/cropsci1995.0011183X003500050023x

Gitleson AA, Gritz Y, Merzlyak MN (2003) Relationships between leaf chlorophyll content and spectral reflectance and algorithms for nondestructive chlorophyll assessment in higher plant leaves. Journal of Plant Physiology 160: 271-82. doi.org/10.1078/0176-1617-00887

McClung CR 2006. Plant circadian rhythms. Plant Cell 18: 792-803. doi.org/10.1105/tpc. 106.040980

Oberbauer SF, Starr G (2002) The role of anthocyanins for photosynthesis of Alaskan arctic evergreens during snowmelt. Advances in Botanical Research 37: 129-45. doi.org/10.1016/S0065-2296(02)37047-2

Patankar R, Mortazavi B, Oberbauer SF, Starr G (2013) Diurnal patterns of gas-exchange and metabolic pools in tundra plants during three phases of the arctic growing season. Ecology and Evolution 3: 375-88. doi.org/10.1002/ece3.467

Starr G, Oberbauer SF, Ahlquist LE (2008) The photosynthetic response of Alaskan tundra plants to increased season length and soil warming. Arctic Antarctic, and Alpine Research 40: 181-91. doi. org/10.1657/1523-0430(06-015)[STARR]2.0.CO;2

Starr G, Oberbauer SF, Pop E (2000) Effects of lengthened growing season and soil warming on the phenology and physiology of Polygonum bistorta. Global Change Biology 6: 357-69. doi.org/10.1046/j.13652486.2000.00316.x

Steane DA, Potts BM, McLean E, Collins L, Prober MS, Stock WD, Vaillancourt RE, Byrne M (2015) Genome-wide scans reveal cryptic population structure in a dry-adapted eucalypt. Tree Genetics \& Genomes 11: 2500-13. doi.org/10.1007/s11295-015-0864-z 
This text is taken from Researching functional ecology in Kosciuszko National Park, edited by Hannah Zurcher, Chia Ming-Dao, Michael Whitehead and Adrienne Nicotra, published 2017 by ANU eView, The Australian National University, Canberra, Australia. dx.doi.org/10.22459/RFEKNP.11.2017.04 